\title{
Ginga: an open-source astronomical image viewer and toolkit
}

\author{
Eric Jeschke $e^{\ddagger *}$ \\ http: //www . youtube. com/watch?v=nZKy_nYUxCs
}

\begin{abstract}
Ginga is a new astronomical image viewer written in Python. It uses and inter-operates with several key scientific Python packages: NumPy, Astropy, and SciPy. A key differentiator for this image viewer, compared to oldergeneration FITS viewers, is that all the key components are written as Python classes, allowing for the first time a powerful FITS image display widget to be directly embedded in, and tightly coupled with, Python code.

We call Ginga a toolkit for programming FITS viewers because it includes a choice of base classes for programming custom viewers for two different modern widget sets: Gtk and Qt, available on the three common desktop platforms. In addition, a reference viewer is included with the source code based on a plugin architecture in which the viewer can be extended with plugins scripted in Python. The code is released under a BSD license similar to other major Python packages and is available on GitHub.

Ginga has been introduced only recently as a tool to the astronomical community, but since SciPy has a developer focus this talk concentrates on programming with the Ginga toolkit. We cover two cases: using the bare image widget to build custom viewers and writing plugins for the existing full-featured Ginga viewer. The talk may be of interest to anyone developing code in Python needing to display scientific image (CCD or CMOS) data and astronomers interested in Python-based quick look and analysis tools.
\end{abstract}

Index Terms-FITS, viewer, astronomical, images, Python, NumPy, SciPy, Astropy

\section{Introduction}

Ginga is a new astronomical image viewer and toolkit written in Python. We call Ginga a toolkit for programming scientific image viewers [Jes12] because it includes a choice of base classes for programming custom viewers for two different modern widget sets: Gtk and Qt, available on the three common desktop platforms (Linux, Mac, and Windows).

Ginga uses and inter-operates with several key scientific Python packages: NumPy, Astropy and SciPy. Ginga will visualize FITS $^{1}$ files as well as other common digital image formats and can operate on any imaging data in NumPy array format. Ginga components are written as Python classes, which allows the image display widget to be directly embedded in, and tightly coupled with, Python code. The display widget supports arbitrary scaling

* Corresponding author: eric@naoj.org

* Subaru Telescope, National Astronomical Observatory of Japan

Copyright $@ 2013$ Eric Jeschke. This is an open-access article distributed under the terms of the Creative Commons Attribution License, which permits unrestricted use, distribution, and reproduction in any medium, provided the original author and source are credited. and panning, rotation, color mapping and a choice of automatic cut levels algorithms.

A reference viewer is included with the Ginga source code based on a plugin architecture in which the viewer can be extended with plugins scripted in Python. Example plugins are provided for most of the features of a "modern" astronomical FITS viewer. Users wishing to develop an imaging program employing Ginga can follow one of two logical development paths: starting from the widget and building up around it, or starting from the reference viewer and customizing it via a plugin.

\section{Getting and installing Ginga}

Ginga is released under a BSD license similar to other major scientific Python packages and is available on GitHub: http: //github.com/ejeschke/ginga . It is a distutils-compatible Python package, and is also available in PyPI. Installing it is as simple as:

pip install ginga

or:

python setup.py install

Use the latter if you have downloaded the latest source as a tarball from http://ejeschke.github.com/ginga or cloned the git repository from https://github.com/ejeschke/ginga.git . The package will be installed as "ginga" and the reference viewer will also be installed as ginga (but located wherever scripts are stored).

Prerequisites and dependences: Ginga will run under Python versions from 2.7 to 3.3. Note that as a minimum you will need to have at least installed numpy and one of the Python Gtk or Qt bindings (e.g. pygtk, pyqt 4). For full functionality you will also need scipy and astropy [Tol13]. Certain features in the reference viewer also be activated if matplotlib is installed.

\section{Part 1: Developing with the Ginga Widget}

When developing with the Ginga toolkit for visualizing FITS files there are two main starting points one might take:

- using only the Ginga widget itself, or

- starting with the full-featured reference viewer that comes with Ginga and customize it for some special purpose.

The first way is probably best for when the developer has a custom application in mind, needs a bare-bones viewer or wants

1. Flexible Image Transport System--the current standard for archiving and exchanging astronomical data as files. 
to develop an entirely new full-featured viewer. The second way is probably best for end users or developers that are mostly satisfied with the reference viewer as a general purpose tool and want to add some specific enhancements or functionality. Because the reference viewer is based on a flexible plugin architecture this is fairly easy to do. In this paper we address both of these approaches.

First, let's take a look at how to use the "bare" Ginga FITS viewing widget by itself. The Fits ImageZoom widget handles image display, scaling (zooming), panning, manual cut levels, auto cut levels with a choice of algorithms, color mapping, transformations, and rotation. Besides the image window itself there are no additional GUI (Graphical User Interface) components and these controls are handled programatically or directly by keyboard and mouse bindings on the window. Developers can enable as many of the features as they want, or reimplement them. The user interface bindings are configurable via a pluggable Bindings class, and there are a plethora of callbacks that can be registered, allowing the user to create their own custom user interface for manipulating the view.

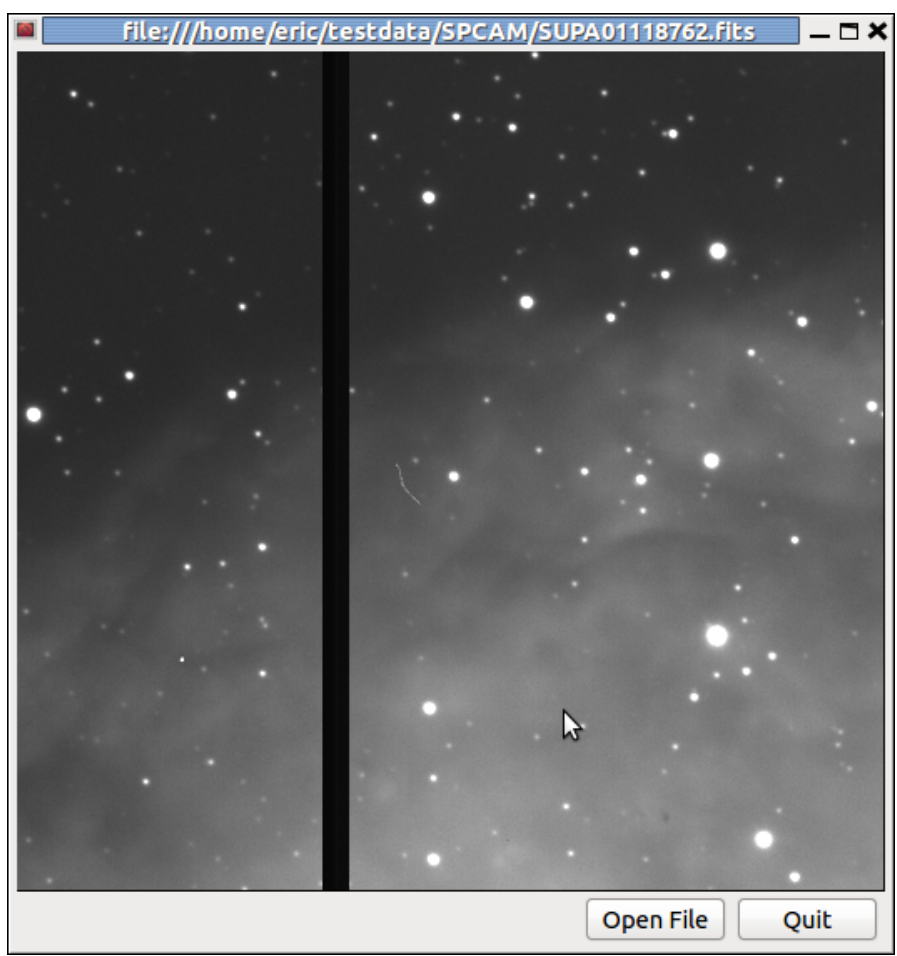

Fig. 1: A simple, "bare bones" FITS viewer written in Qt.

Listing 1 shows a code listing for a simple graphical FITS viewer using this widget (screenshot in Figure 1) written in around 100 or so lines of Python. It creates a window containing an image view and two buttons. This example, included with the Ginga package, will open FITS files dragged and dropped on the image window or via a dialog popped up when clicking the "Open File" button.

Looking at the constructor for this particular viewer, you can see where we create a Fits ImageZoom object. On this object we enable automatic cut levels (using the 'zscale' algorithm), auto zoom to fit the window and set a callback function for files dropped on the window. We extract the user-interface bindings with get_bindings(), and on this object enable standard user interactive controls for panning, zooming, cut levels and simple transformations (flip $\mathrm{x} / \mathrm{y}$ and swap axes). We then extract the platform-specific widget (Qt-based, in this case) using get_widget () and pack it into a Qt container along with a couple of buttons to complete the viewer.

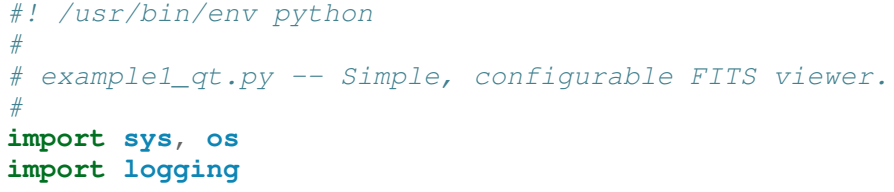




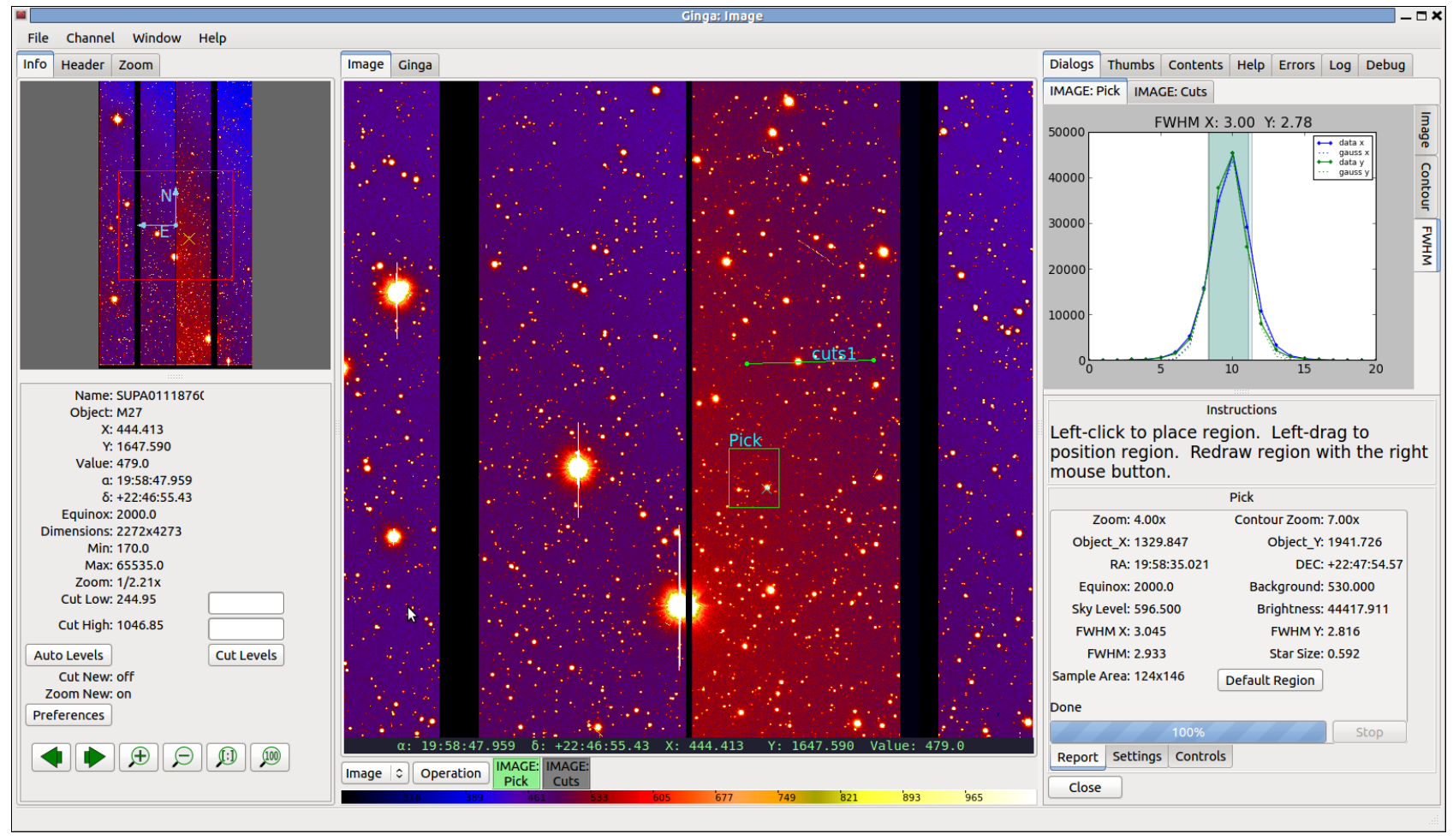

Fig. 3: The Ginga reference viewer (Qt version), with some plugins active.

has it open, and does not capture the mouse actions unless the channel it is operating on is selected. Thus one can have two different Pick operations going on concurrently on two different channels, for example, or a Pick operation in a camera channel, and a Cuts (line cuts) operation on a spectrograph channel. Figure 5 shows an example of the Pick local plugin occupying a notebook tab.

\section{Anatomy of a Local Ginga Plugin}

Let's take a look at a local plugin to understand the API for interfacing to the Ginga shell. In Listing 2, we show a stub for a local plugin.

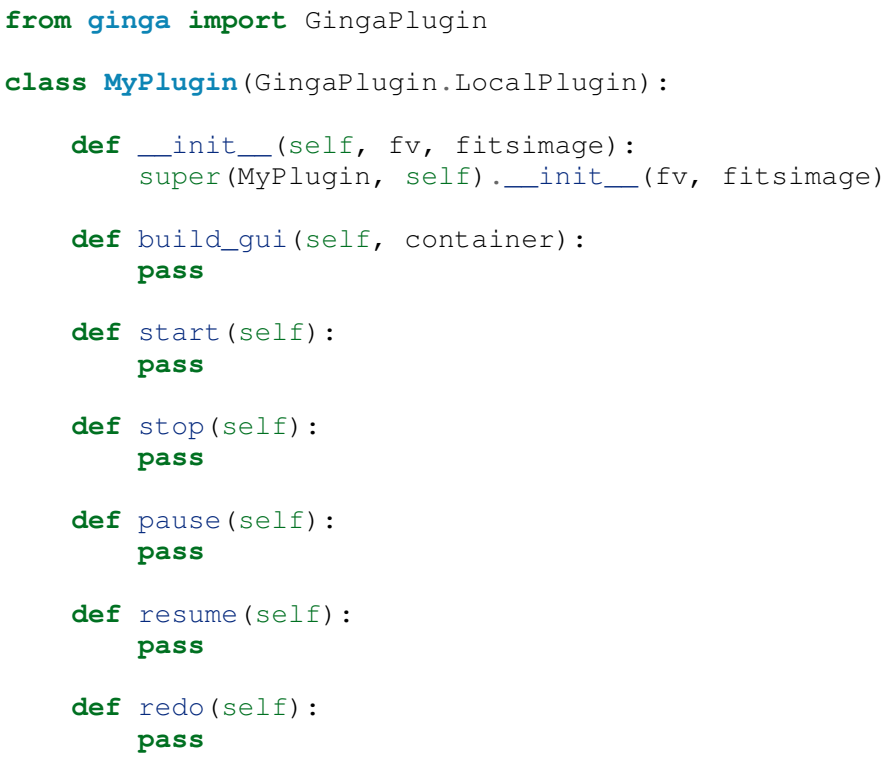

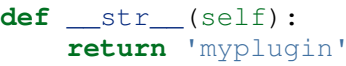

The purpose of each method is as follows.

init__(self, fv, fitsimage): This method is called when the plugin is loaded for the first time. $f v$ is a reference to the Ginga shell and fitsimage is a reference to the Fits ImageCanvas object associated with the channel on which the plugin is being invoked. You need to call the superclass initializer and then do any local initialization.

build_gui(self, container): This method is called when the plugin is invoked. It builds the GUI used by the plugin into the widget layout passed as container. This method may be called many times as the plugin is opened and closed for modal operations. The method may be omitted if there is no GUI for the plugin.

start(self): This method is called just after build_gui() when the plugin is invoked. This method may be called many times as the plugin is opened and closed for modal operations. This method may be omitted.

stop(self): This method is called when the plugin is stopped. It should perform any special clean up necessary to terminate the operation. The GUI will be destroyed by the plugin manager so there is no need for the stop method to do that. This method may be called many times as the plugin is opened and closed for modal operations. This method may be omitted if there is no special cleanup required when stopping.

pause (self): This method is called when the plugin loses focus. It should take any actions necessary to stop handling user interaction events that were initiated in start () or resume (). This method may be called many times as the plugin is focused or defocused. The method may be omitted if there is no user event handling to disable. 


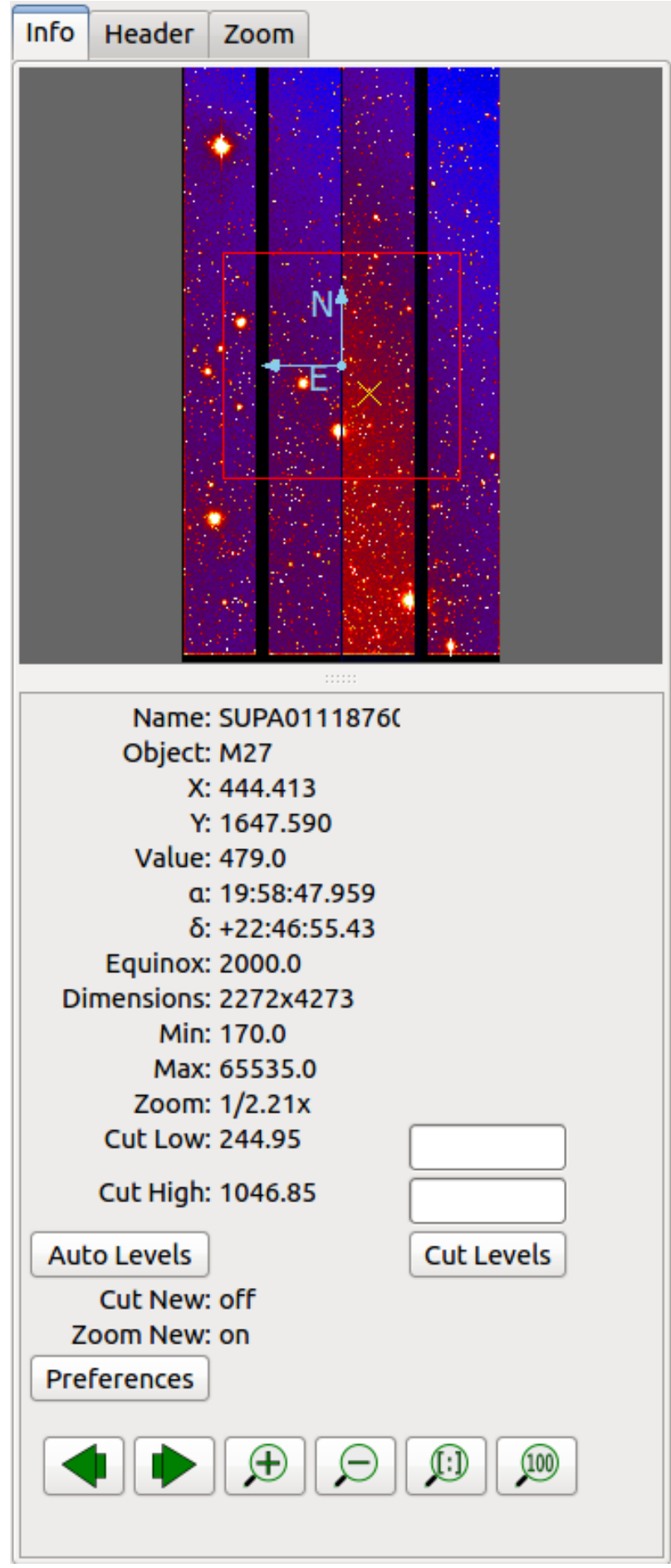

Fig. 4: Two global plugins: Pan (top) and Info (bottom), shown sharing a tab.

resume ( $\operatorname{sel} f)$ : This method is called when the plugin gets focus. It should take any actions necessary to start handling user interaction events for the operations that it does. This method may be called many times as the plugin is focused or defocused. The method may be omitted if there is no user event handling to enable.

redo ( $\operatorname{self}$ ): This method is called when the plugin is active and a new image is loaded into the associated channel. It can optionally redo the current operation on the new image. This method may be called many times as new images are loaded while

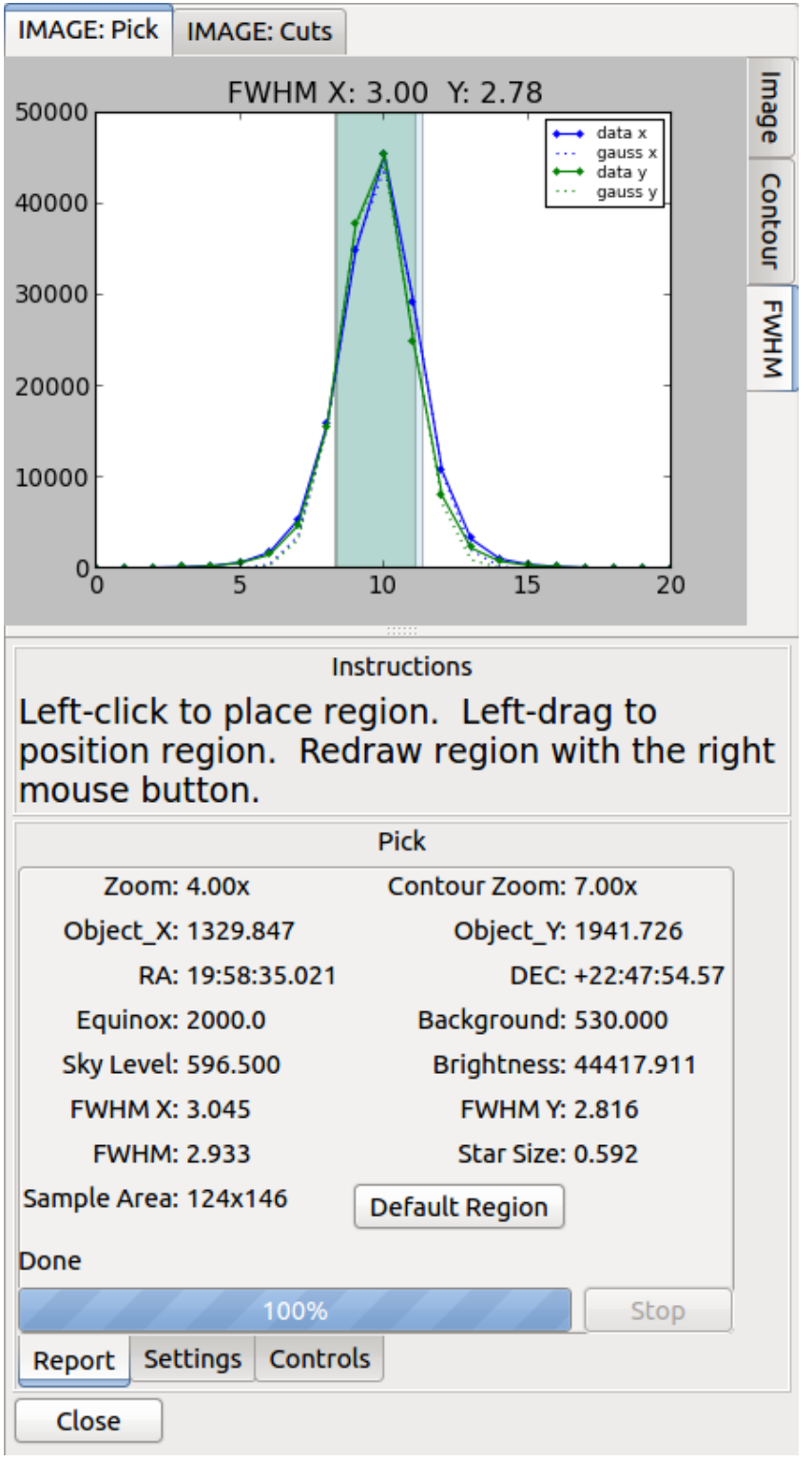

Fig. 5: The Pick local plugin, shown occupying a tab.

the plugin is active. This method may be omitted.

\section{Putting it All Together: The Ruler Plugin}

Finally, in Listing 3 we show a completed plugin for Ruler. The purpose of this plugin to draw triangulation (distance measurement) rulers on the image. For reference, you may want to refer to the ruler shown on the canvas in Figure 2 and the plugin GUI shown in Figure 6.

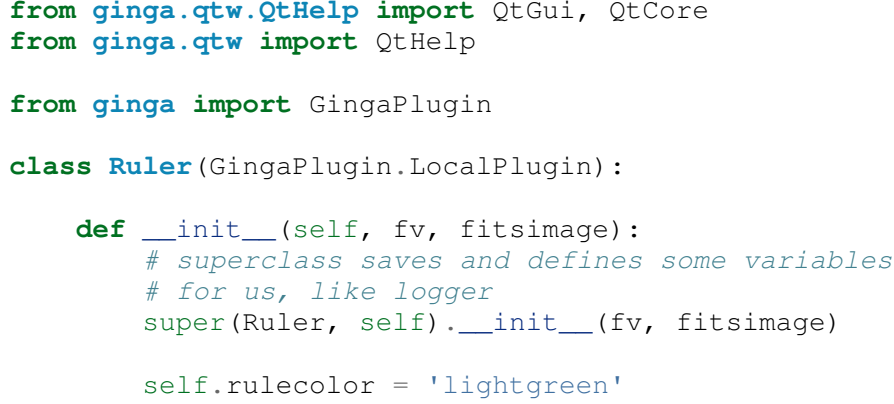




\begin{tabular}{l}
\hline IMAGE: Ruler \\
$\begin{array}{l}\text { Instructions } \\
\text { Draw (or redraw) a line with the right mouse } \\
\text { button. Display the Zoom tab to precisely } \\
\text { see detail. } \\
\text { Units: arcmin } \\
\text { Close }\end{array}$ \\
\hline
\end{tabular}

Fig. 6: The Ruler local plugin GUI, shown occupying a tab.

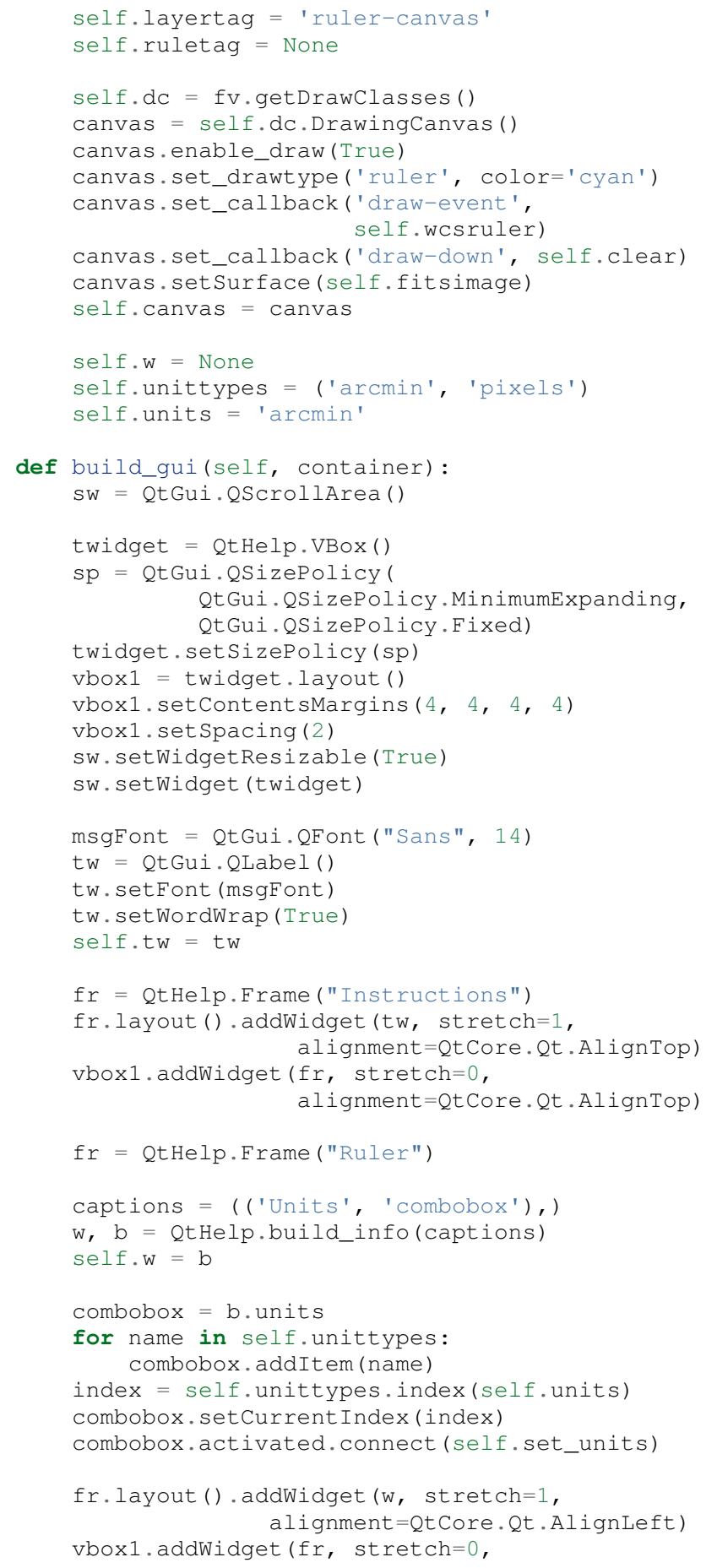

alignment=QtCore. Qt. AlignTop)

btns $=$ QtHelp.HBox ()

layout = btns.layout ()

layout.set Spacing (3)

\#btns.set_child_size (15, -1)

btn = QtGui.QPushButton ("Close")

btn.clicked.connect (self.close)

layout.addWidget (btn, stretch=0, alignment $=$ Qt Core. Qt . AlignLeft)

vbox1.addWidget (btns, stretch=0, alignment=QtCore.Qt. AlignLeft)

container.addWidget ( $\mathrm{sw}$, stretch=1)

def set units (self):

index $=$ self.w.units.currentIndex ()

units = self.unittypes[index]

self.canvas.set_drawtype('ruler', color='cyan',

self.redo() units=units)

return True

def close(self):

chname = self.fv.get channelName ( self.fitsimage)

self.fv.stop_operation_channel(chname, $\operatorname{str}(\operatorname{sel} f))$

return True

def instructions(self): self.tw.setText ("Draw (or redraw) a line " "with the right mouse " "button. Display the " "Zoom tab to precisely " "see detail.")

self.tw.show()

def start(self):

self.instructions()

\# start ruler drawing operation try:

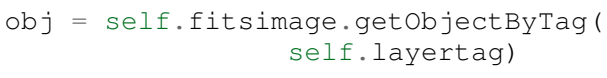

except KeyError:

\# Add ruler layer

self.fitsimage.add (self.canvas, tag=self. layertag)

self.canvas.deleteAllobjects()

self.resume ()

def pause(self): self.canvas.ui_setActive (False)

def resume(self): self.canvas.ui_setActive (True) self.fv.showstatus("Draw a ruler with "

"the right mouse button")

def stop(self):

\# remove the canvas from the image,

\# this prevents us from getting draw events

\# when we are inactive

try:

self.fitsimage. deleteobjectByTag(

except : self.layertag)

\section{pass}

self.fv.showstatus(" )

def redo(self):

\# get the ruler object on the canvas obj = self.canvas.getobjectByTag( 


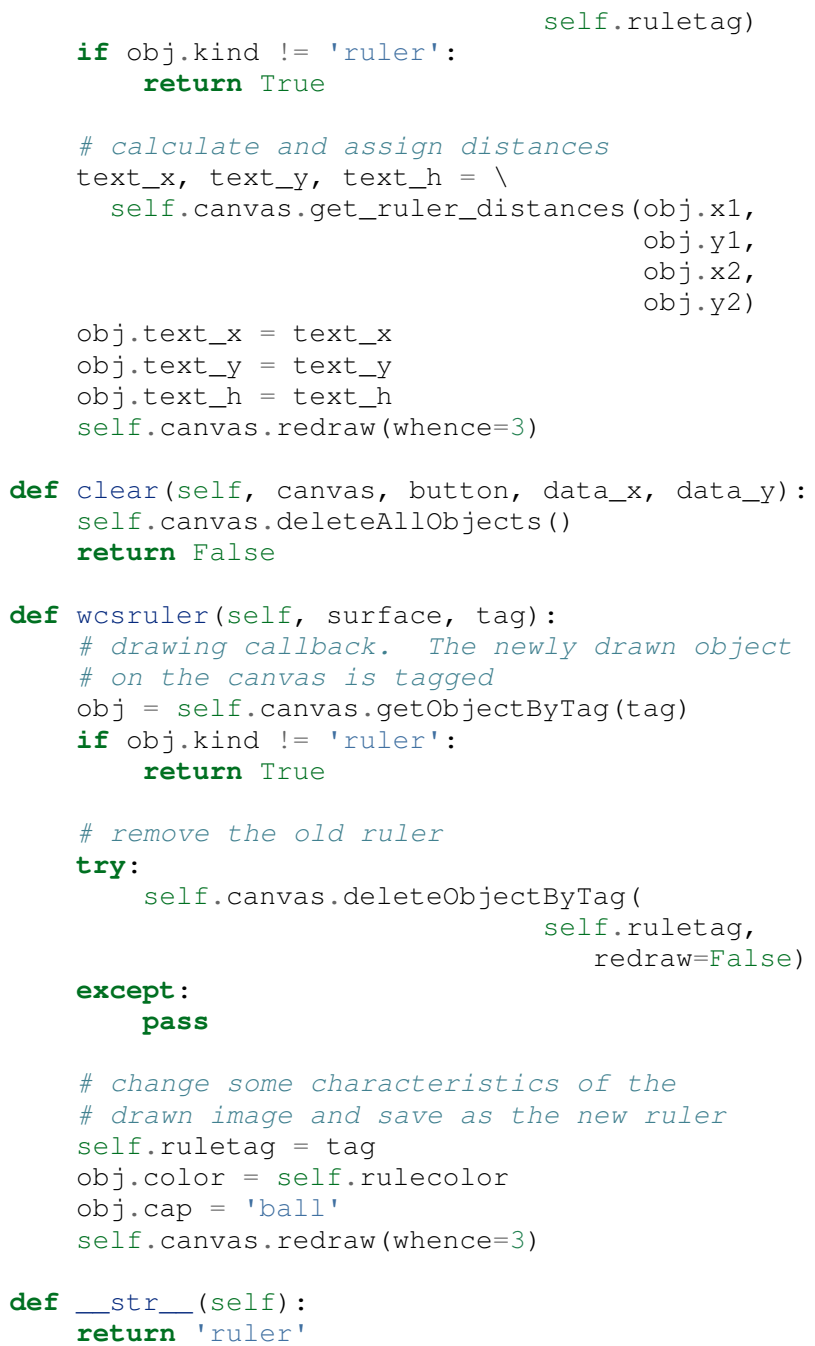

This plugin shows a standard design pattern typical to local plugins. Often one is wanting to draw or plot something on top of the image below. The Fits ImageCanvas widget used by Ginga allows this to be done very cleanly and conveniently by adding a DrawingCanvas object to the image and drawing on that. Canvases can be layered on top of each other in a manner analogous to "layers" in an image editing program. Since each local plugin maintains it's own canvas, it is very easy to encapsulate the logic for drawing on and dealing with the objects associated with that plugin. We use this technique in the Ruler plugin. When the plugin is loaded (refer to _ init_ () method), it creates a canvas, enables drawing on it, sets the draw type and registers a callback for drawing events. When start () is called it adds that canvas to the widget. When stop ( ) is called it removes the canvas from the widget (but does not destroy the canvas). pause () disables user interaction on the canvas and resume () reenables that interaction. redo() simply redraws the ruler with new measurements taken from any new image that may have been loaded. In the __init__ () method you will notice a setSurface () call that associates this canvas with a Fits Image-based widget--this is the key for the canvas to utilize WCS information for correct plotting. All the other methods shown are support methods for doing the ruler drawing operation and interacting with the plugin GUI.

The Ginga package includes a rich set of classes and there are also many methods that can be called in the shell or in the Fits ImageCanvas object for plotting or manipulating the view. Length constraints do not permit us to cover even a portion of what is possible in this paper. The best way to get a feel for these APIs is to look at the source of one of the many plugins distributed with Ginga. Most of them are not very long or complex. In general, a plugin can include any Python packages or modules that it wants and programming one is essentially similar to writing any other Python program.

\section{Writing a Global Plugin}

This last example was focused on writing a local plugin. Global plugins employ a nearly identical API to that shown in Listing 2 , except that the constructor does not take a fitsimage parameter, because the plugin is expected to be active across the entire session, and is not associated with any particular channel. build_gui() and start() are called when the Ginga shell starts up, and stop() is never called until the program terminates ${ }^{2}$. pause () and resume () can safely be omitted because they should never be called. Like local plugins, build_gui () can be omitted if there is no GUI associated with the plugin. Take a look at some of the global plugins distributed with the viewer for more information and further examples. The IRAF plugin, which handles IRAF/ginga interaction similarly to IRAF/ds9, is an example of a plugin without a GUI.

\section{Conclusion}

The Ginga FITS viewer and toolkit provides a set of building blocks for developers wishing to add FITS image visualization to their Python-based application, or end users interested in a Python-scriptable, extensible viewer. Two avenues of development are possible: a "blue sky" approach by using a flexible FitsImageCanvas display widget and building up around that, or by starting with the plugin-based reference viewer and customizing by modifying or writing new plugins. In either case, the software can be targeted to two different widget sets (Gtk and Qt) across the common desktop platforms that Python is available on today. The code is open-sourced under a BSD license and is available via the GitHub code repository or via PyPI.

Future plans for Ginga mostly center around the development of some additional plugins to enhance capabilities. Ideas suggested by users include:

- mosaicking of images

- simple, user-customizable pipelines for handling flat fielding, bias frames, dark frame subtraction, bad pixel masking, etc.

- improving the set of graphical plotting elements

- semi-transparent colored overlays, for showing masks, etc.

- improving PDF and postscript output options

\section{RefERENCES}

[Jes12] E. Jeschke, T. Inagaki and R. Kackley. A next-generation open-source toolkit for FITS file image viewing, Software and Cyberinfrastructure for Astronomy II, Proceedings SPIE, 8451(1), 2012.

[Tol13] E. Tollerud and P. Greenfield and T. Robitaille. The Astropy Project: A Community Python Library for Astrophysics, ADASS XXII, ASP Conf Ser., TBD:(in press), 2013.

2. Unless the user reloads the plugin. Most plugins in Ginga can be dynamically reloaded using the Debug plugin, which facilitates debugging tremendously, since Ginga itself does not have to be restarted, data does not have to be reloaded, etc. 